\title{
Infarto agudo de miocardio asociado al consumo de sildenafil. Aportación de caso y revisión de la literatura
}

\author{
Velásquez López JG*, Agudelo Restrepo CA**, Yepes Gómez D***, Uribe Trujillo CA* \\ *Servicio de Urología Hospital Pablo Tobón Uribe y Universidad CES. **Departamento de Medicina Interna. \\ Universidad Pontificia Bolivariana. ***Unidad de Cuidados Intensivos. Clinica Medellin. \\ Hospital Pablo Tobón Uribe. Medellín (Colombia). \\ Actas Urol Esp. 2007;31(1):52-57
}

\section{RESUMEN \\ INFARTO AGUDO DE MIOCARDIO ASOCIADO AL CONSUMO DE SILDENAFIL. APORTACIÓN DE CASO Y REVISIÓN DE LA LITERATURA.}

La disfunción eréctil afecta aproximadamente a 30 millones de personas en los Estados Unidos. El Sildenafil fue el primer inhibidor de la 5-Fosfodiesterasa (5-PDE) aprobado por la FDA para la disfunción eréctil. Desde entonces su uso se ha incrementado notablemente. Este medicamento tiene varios efectos adversos generales como cefalea, enrojecimiento facial, congestión nasal, alteraciones visuales, dispepsia, diarrea, vértigo y rash; fenómenos cardiovasculares incluyen infarto de miocardio, taquicardia ventricular, angina y muerte. Las guías de manejo con respecto al uso del Sildenafil en pacientes con enfermedad coronaria, advierten sobre los riesgos cardiovasculares de la medicación. Se han reportado hasta el momento una cifra incierta de infartos de miocardio en pacientes tomando la medicación, hecho que ha obligado al productor a introducir una advertencia en la etiqueta. Nosotros reportamos un caso de IAM luego de la ingesta de $100 \mathrm{mg}$ de sildenafil, sin relación sexual posterior y sin consumo de nitratos, pero con enfermedad arterioesclerótica difusa documentada por coronariografía.

Palabras clave: Sildenafil. Infarto agudo de miocardio. Enfermedad coronaria. Impotencia. Agentes vasodilatadores. Disfunción eréctil.

\section{ABSTRACT \\ ACUTE MYOCARDIAL INFARCTION ASSOCIATED TO THE SILDENAFIL CONSUMPTION. A CASE REPORT AND REVIEW OF THE LITERATURE.}

Erectile dysfunction affects more than 30 million men in The United States. Since the FDA approved the use of Sildenafil, prescription of this medication has been raising. Adverse events of Sildenafil includes: fatigue, dyspnea, and hypotension. Reported adverse cardiac events associated with the medication use include myocardial infarction, ventricular tachycardia, angina and death, raising concerns about the safety of this agent in patients with coronary artery disease. Published guidelines regarding the management of cardiac patients with erectile dysfunction suggest that Sildenafil may be hazardous in patients with ischemic heart disease. In patients using Sildenafil, myocardial infarctions have been reported to the Food and Drug Administration. Now, we report a patient with myocardial infarction after taking $100 \mathrm{mg}$ of Sildenafil without sexual activity.

Keywords: Sildenafil. Acute myocardial infarction. Coronary disease. Ipotence. Vasodilator agents. Erectile dysfunction. 
$\mathrm{E}_{\mathrm{r}}^{1}$ sildenafil es el primer inhibidor de la 5Fosfodiesterasa (5-PDE) aprobado para el manejo de la disfunción eréctil (DE), inicialmente por la Food and Drugs Administration (FDA) en los Estados Unidos y luego por la European Medicines Evaluation Agency ${ }^{1}$. La alta prevalencia de pacientes adultos y de edad avanzada, asociado a la mayor sobrevida que tienen hoy en día los pacientes con enfermedades anteriormente de alta mortalidad, hacen que la prevalencia de entidades como la DE sea elevada. Al ser uno de los métodos más efectivos de tratamiento, el consumo de esta clase de droga ha logrado llegar a cantidades exponenciales. Para finales del 2000 se reportaron más de 10 millones de personas que recibieron tratamiento con sildenafil ${ }^{7,17}$. Entre los efectos adversos del sildenafil se describen la cefalea, el enrojecimiento facial (flushing), congestión nasal, alteraciones visuales, dispepsia, diarrea, vértigo y $\operatorname{rash}^{2,15,17,19}$. Nosotros aportamos un caso de infarto agudo de miocardio (IAM), asociado al consumo de sildenafil en una dosis de $100 \mathrm{mg}$.

\section{CASO CLÍnICO}

Se trata de un paciente masculino de 70 años, quien ingresó en el servicio de urgencias del Hospital Pablo Tobón Uribe, con cuadro de aproximadamente una hora de evolución consistente en opresión torácica, malestar general, astenia y diaforesis; que iniciaron después de haber ingerido $100 \mathrm{mg}$ de sildenafil, niega ingesta de otro estimulante sexual o cocaína y sin relación sexual después de su consumo. El paciente como único antecedente clínico sufría de hipertensión arterial, controlada farmacológicamente y niega episodios previos de angina o consumo de nitratos. El examen clínico y sus signos vitales eran normales; sin embargo, después de la valoración inicial presenta paro cardiorrespiratorio secundario a fibrilación ventricular con respuesta a una única desfibrilación de 200 joules.

El electrocardiograma inicial (Fig. 1) demostró elevación del segmento ST en las derivadas de la cara inferior (II, III y aVF) y de la cara anterior (V2-V4) con cambios recíprocos en aVL, sin demostrarse extensión electrocardiográfica a ventrículo derecho.

Las enzimas cardíacas al ingreso revelaron una creatinfosfokinasa (CK) de170 y una creatinfosfokinasa fracción MB (CK-MB) de 6. Los electrolitos, las pruebas de coagulación y los conteos de células sanguíneas fueron normales.

El manejo inicial se realizó con aspirina $100 \mathrm{mg}$, lovastatina $40 \mathrm{mg}$ cada día, metoprolol $25 \mathrm{mg}$ cada 12 horas, enoxaparina $60 \mathrm{mg}$ cada 12 horas, oxígeno a $3 \mathrm{lt} / \mathrm{min}$ y estreptoquinasa $1 ` 500.000$ unidades administradas en 30 minutos. No se demostraron cambios secundarios a reperfusión. El paciente fue trasladado a la unidad de cuidados intensivos, donde se documentaron durante las primeras horas de evolución, episodios de bloqueo A-V completo con resolución espontánea.

El EKG tomado a las 24 horas de evolución reveló $\mathrm{QS}$ en cara inferior y una progresión tardía de la onda $\mathrm{R}$ en la cara anterior. El seguimiento enzimático demostró aumento de CK y de la fracción MB a las 6 horas (4476 y 165) y a las 12 horas (3839 y 136).

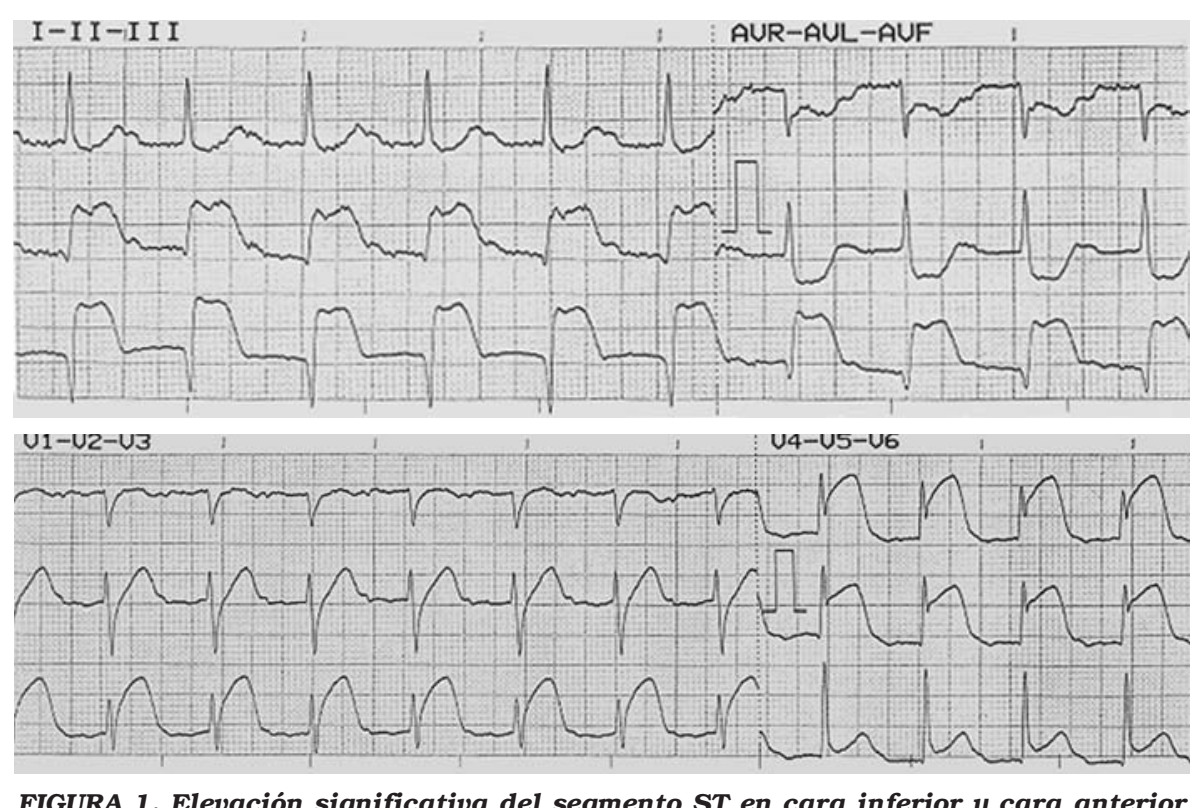

1. Elevación significativa del segmento ST en cara inferior y cara anterior en EKG tomado al ingreso del paciente al servicio de urgencias. 
Al día siguiente se realizó una coronariografía que mostró enfermedad difusa de la arteria descendente anterior con lesión en el tercio distal del $50 \%$ y lesión del $40 \%$ en el tercio proximal de la primera rama diagonal. La arteria circunfleja tenía una lesión del $50 \%$ en el tercio medio y enfermedad difusa de sus ramas obtusas marginales. La arteria coronaria derecha presentaba una lesión irregular sugestiva de trombo parcialmente resuelto produciendo estenosis máxima del $50 \%$; distalmente la arteria descendente posterior presentaba dos lesiones del 40\% (Figs. 2 y 3).

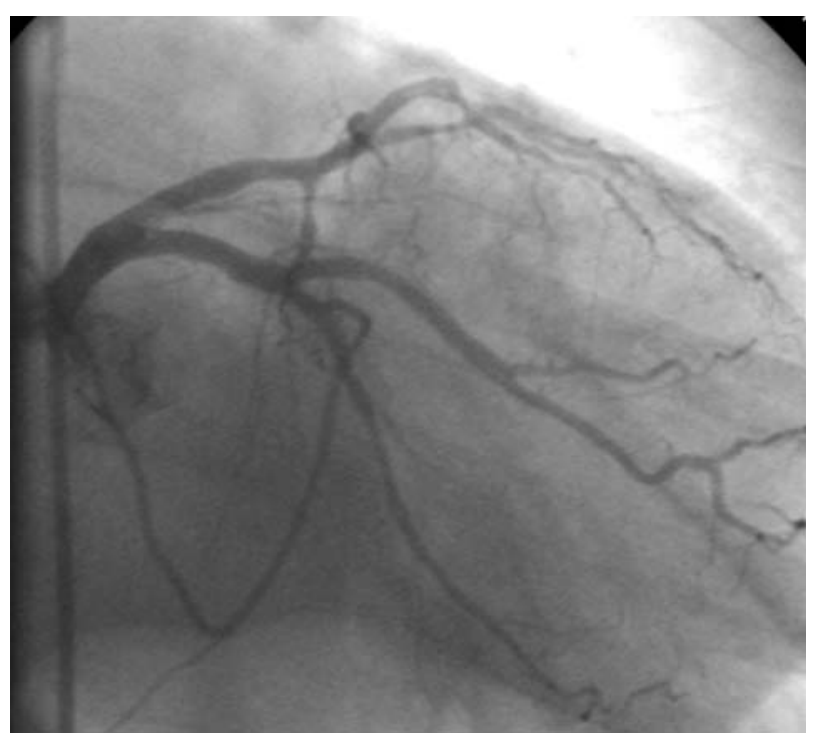

FIGURA 2. Enfermedad ateroesclerótica difusa de las arterias descendente anterior, circunfleja y sus ramas.

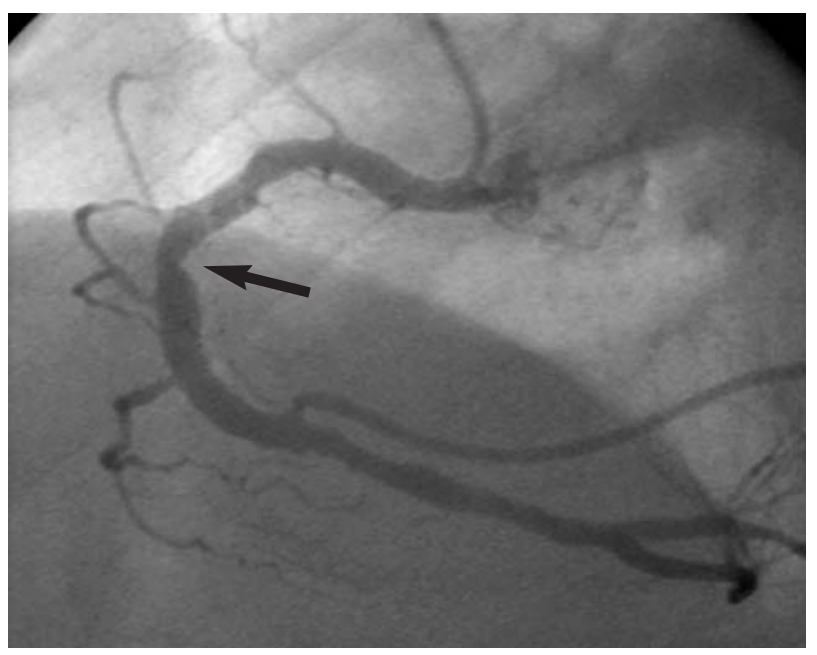

FIGURA 3. Arteria coronaria derecha con imagen sugestiva de trombo parcialmente resuelto en el tercio medio.
El paciente evolucionó satisfactoriamente sin complicaciones posteriores y libre de dolor. Fue dado de alta para seguimiento ambulatorio.

\section{DISCUSIÓN}

La DE es una entidad que afecta a más de 30 millones de hombres sólo en Estados Unidos ${ }^{17,23}$. La prevalencia mundial de esta entidad para 1995 fue de 152 millones de personas afectadas, pero se esperan para el 2025 alrededor de 322 millones de personas con $\mathrm{DE}^{6}$, en relación con el envejecimiento de la población. La prevalencia reportada de $\mathrm{DE}$ en hombres mayores de 40 años es igual o mayor al 30\%. En EEUU el estudio Massachussets Male Aging Study (MMAS) realizado en Massachussets entre 1987 y 1989, demostró que el $52 \%$ de los hombres que habitan en ciudades y pueblos próximos a Boston presentaban $\mathrm{DE}^{4}$. En Latinoamérica los estudios muestran una incidencia similar; en el estudio DENSA por Morillo et al. se reportó una tasa de DE del 53,4\% entre hombres mayores de 40 años en Colombia, Venezuela y Ecuador ${ }^{5}$.

La enzima 5-PDE es una de las 11 isoenzimas conocidas en el organismo humano. Se encuentra localizada en la célula del músculo liso y predomina en el cuerpo cavernoso del pene, sin embargo se ha reportado su presencia en otros tejidos como en el cardiomiocito de caninos ${ }^{8}$. Esta enzima es la encargada del desdoblamiento del 3',5'-guanosin monofosfato cíclico (GMPc) en 5' guanosin monofosfato (5'GMP). El GMPc a través de una protein kinasa específica permite la salida del calcio intracelular, llevando así a un estado de relajación y vasodilatación, con aumento del flujo sanguíneo, esencial para una adecuada erección ${ }^{2}$. El sildenafil actúa inhibiendo de manera específica la 5-PDE, con relativa poca reacción cruzada con otras PDE. Su estructura química es similar a la del GMPc y logra inhibir la unión de la 5-PDE con el GMPc (sitio catalítico), produciendo una acumulación de GMPc en el tejido eréctil ${ }^{7}$. Dos agentes adicionales de esta misma clase han sido desarrollados en años recientes el tadalafil y el vardenafil.

Se ha demostrado la eficacia terapéutica del sildenafil en el tratamiento de la DE orgánica o situacional, y secundaria a prostatectomía radical, radioterapia, diabetes mellitus, lesión de medula 
espinal y enfermedad de parkinson $3,17,19,21$. Investigaciones clínicas recientes en humanos y modelos animales también han demostrando que las propiedades vasodilatadoras y el desencadenante bioquímico resultante de su acción tienen efectos benéficos en otras entidades como la hipertensión pulmonar ${ }^{7,9}$, la insuficiencia cardíaca congestiva ${ }^{12}$ y la disfunción endotelial ${ }^{7,24}$. Se ha sugerido un efecto protector en la lesión por repercusión del sistema nervioso central, el hígado y otros órganos ${ }^{7}$. En modelos animales se ha visto una acción cardioprotectora secundaria a un efecto de preacondicionamiento miocárdico que podría disminuir el riesgo de $\mathrm{IAM}^{7,25}$.

La aparición de cefalea, congestión nasal y enrojecimiento facial, sugiere que esta droga no sólo actúa a nivel de los cuerpos cavernosos. La redistribución del flujo sanguíneo, secundaria a una disminución rápida de la resistencia vascular periférica puede alterar la perfusión coronaria normal y producir isquemia miocárdica ${ }^{1,3} . \mathrm{El}$ daño vascular coronario pre-existente aumenta la susceptibilidad a esta clase de eventos ${ }^{1}$. El uso de nitratos produce vasodilatación universal por aumento de los niveles de oxido nítrico (ON), potenciada por el sildenafil, llevando a una importante caída de la presión arterial ${ }^{19}$. La subsiguiente redistribución del flujo sanguíneo arterial puede resultar en un fenómeno de "robo" sanguíneo coronario y desencadenar un evento cardíaco isquémico ${ }^{15,16}$. Por ello la terapia con nitratos es una contraindicación absoluta para el consumo o prescripción de sildenafil ${ }^{17,23}$.

Los análisis de las tasas de IAM y muerte en pacientes que han ingerido sildenafil ${ }^{13-18}$ son controversiales, unos defienden su seguridad cardíaca mientras que otros apoyan su efecto causal de isquemia miocárdica por los mecanismos arriba mencionados. Las guías para el uso de sildenafil en pacientes con enfermedad cardiovascular publicadas en el American Journal of Cardiology ${ }^{10}$, recomiendan una clasificación previa de los pacientes antes de su uso y recomiendan estratificarlos como de bajo, intermedio o alto riesgo; sólo en los de alto riesgo su uso requiere evaluación cardiovascular por el elevado riesgo de lesión isquémica ${ }^{3}$, en los demás se considera una droga segura. Apoyando esto último Herrmann et al. lograron demostrar en humanos con enfermedad coronaria estable que el sildenafil no tiene efectos cardiovasculares adversos directos en estos pacientes ${ }^{20}$.

El compromiso producido por la ateroesclerosis y la disfunción endotelial se ha propuesto como el mecanismo subyacente que explica la alta tasa de asociación entre la DE y la enfermedad coronaria. Montorsi et al. han sugerido el compromiso ateroesclerótico difuso con manifestaciones variables según el calibre de la arteria afectada (Hipótesis del tamaño de la arteria), donde la DE es frecuentemente una manifestación que precede la enfermedad coronaria debido al menor tamaño de la arteria cavernosa en comparación con las arterias coronarias ${ }^{11}$. Datos clínicos muestran que pacientes con DE tienen una incidencia del $20 \%$ de presentar enfermedad arterial coronaria concomitante, mientras que el $60 \%$ de los pacientes con enfermedad arterial coronaria presentan $\mathrm{DE}$ al momento del diagnóstico.

Varios son los casos de eventos isquémicos asociados al consumo de sildenafil 1,15,22,28. La FDA reportó más de 100 muertes en pacientes a quienes se les prescribió sildenafil durante los primeros meses de venta al público ${ }^{17,21}$, de las cuales la mitad correspondieron a eventos cardíacos y menos de la cuarta parte a IAM, y según un caso posterior (13 meses después de la introducción del medicamento) la cifra de muertes podría ascender a más de 500 pacientes $^{21}$; sin embargo se desconoce si estos eventos fueron causados por el medicamento o son secundarios a la presencia de enfermedad cardiovascular subyacente. Un caso posterior del mismo organismo no encontró un número mayor de muertes por IAM en usuarios de sildenafil que en las espe$\operatorname{radas}^{27}$. Shakir et al. demostraron, una menor mortalidad en una cohorte de pacientes usando sildenafil en comparación con la población general del Reino Unido, aunque se cree que la observación podría estar sesgada por subregistro de los eventos ${ }^{26}$. Lo cierto es que la incidencia actual de este tipo de eventos es desconocida por la gran cantidad de público que tiene acceso a la droga, subregistro de reacciones fatales adversas y por falta de estudios farmacoepidemiológicos serios que evalúen la incidencia de éstos eventos en los consumidores $^{21}$. 
El American College of Cardiology y la American Heart Association se han pronunciado acerca de los potenciales efectos adversos del sildenafil (muerte súbita, infarto agudo de miocardio, hemorragia cerebral, ataque isquémico transitorio $\mathrm{y}$ arritmias ventriculares), la casa farmacéutica ha introducido la advertencia en la etiqueta del producto y se ha promovido la prescripción cuidadosa de este medicamento en pacientes con antecedente de isquemia coronaria, fallo cardíaco, baja presión arterial, ingesta de múltiples antihipertensivos o drogas que afecten el metabolismo del sildenafil $^{23}$. Otros autores como Cohen han recomendado el uso de dosis bajas iniciales (test de la "baja dosis" inicial) como una medida efectiva y más útil que la prueba de esfuerzo o la monitorización continua de la presión arterial después del consumo del sildenafil, para evitar reacciones adversas fatales (eventos isquémicos, reacción de la primera dosis, hipotensión, etc.) con dosis mayores del medicamento ${ }^{21}$.

En conclusión, hemos presentado el caso de un paciente con IAM después de la ingesta de sildenafil, sin uso previo de nitratos y sin hipotensión documentada, con oclusión aguda por trombo en arterias coronarias con evidencia arteriográfica de ateroesclerosis no significativa. Aunque estudios pequeños han sugerido la ausencia de compromiso hemodinámico en las arterias coronarias, se requieren estudios adicionales que busquen explicaciones fisiopatológicas de los casos de IAM asociados al uso de sildenafil cada vez más reportados en la literatura. Mientras nuevos datos son obtenidos se recomienda la vigilancia de los potenciales efectos adversos sobre la circulación coronaria del sildenafil al prescribirlo, principalmente en pacientes con riesgo de enfermedad coronaria o que ingieren medicamentos vasodilatadores como los nitratos. Se sugiere, advertir de manera clara al paciente acerca de los riesgos y beneficios con el consumo de esta droga, y en aquellos pacientes de edad avanzada el inicio a bajas dosis.

\section{REFERENCIAS}

1. Feenstra J, van Drie-Pierik RJ, Lacle CF, Stricker BH. Acute myocardial infarction associated with sildenafil. Lancet 1998;352(9132):957-958.

2. Goldstein I, Lue TF, Padma-Nathan H, Rosen RC, Steers WD, Wicker PA. Oral sildenafil in the treatment of erectile dysfunction. Sildenafil Study Group. N Engl J Med. 1998; 338(20):1397-1404.
3. Tran D, Howes LG. Cardiovascular safety of sildenafil. Drug Safety 2003;26(7):453-460.

4. Feldman HA, Goldstein I, Hatzichristou DG, Krane RJ, McKinlay JB. Impotence and its medical and psychosocial correlates: results of the Massachusetts Male Aging Study. J Urol. 1994;151(1):54-61.

5. Morillo LE, Díaz J, Estevez E, Costa A, Méndez H, Dávila H et al. Prevalence of erectile dysfunction in Colombia, Ecuador and Venezuela: a population-based study (DENSA). Int J Impot Res. 2002;14(S2):S10-18.

6. Aytac IA, Mckinlay JB, Krane RJ. The likely worldwide increase in erectile dysfunction between 1995 and 2025 and some possible policy consequences. BJU Internal. 1999; 84(1):50-56.

7. Kukreja RC, Ockaili R, Salloum F, Yin C, Hawkins J, Das A, Xi L. Cardioprotection with phosphodiesterase- 5 inhibition-a novel preconditioning strategy. Journal of Molecular and Cellular Cardiology 2004;36(2):165-173.

8. Senzaki H, Smith CJ, Juang GJ, Isoda T, Mayer SP, Ohler A et al. Cardiac phosphodiesterase 5(cGMP- specific) modulates betaadrenergic signaling in vivo and is downregulated in heart failure. FASEB J 2001;15(10):17181726.

9. Sebkhi A, Strange JW, Phillips SC, Wharton J, Martin R, Wilkins MR. Phosphodiesterase type 5 as a target for the treatment of hypoxia-induced pulmonary hypertension. Circulation 2003;107(25):3230-3235.

10. DeBusk R, Drory Y, Goldstein I, Jackson G, Kaul S, Kimmel SE, et al. Management of sexual dysfunction in patients with cardiovascular disease: recommendations of the Princeton Consensus Panel. Am J Cardiol 2000;86(2): 175-181.

11. Montorsi P, Ravagnani PM, Galli S, Briganti A, Salonia A, Dehò $\mathrm{F}$ et al. Association between erectile dysfunction and coronary artery disease: A case report study. J Sex Med. 2005;2(4):575-582.

12. Bocchi EA, Guimaraes G, Mocelin A, Bacal F, Bellotti G, Ramires JF. Sildenafil effects on exercise, neurohumoral activation, and erectile dysfunction in congestive heart failure. A double-blind, placebo-controlled, randomized study followed by a prospective treatment for erectile dysfunction. Circulation 2002;106(9): 1097-1103.

13. Ockaili R, Salloum F, Hawkins J, Kukreja RC. Sildenafil (Viagra) induces powerful cardioprotective effect via opening of mitochondrial KATP channels in rabbits. Am J Physiol. 2002;283(3):H1263-269.

14. Reffelmann T, Kloner RA. Effects of sildenafil on myocardial infarct size, microvascular function, and acute ischemic left ventricular dilation. Cardiovascular Research 2003;59(2):441-449.

15. Muñiz A, Holstege CP. Acute myocardial infarction associated with sildenafil (viagra) ingestion (correspondence). American Journal of Emergency Medicine 2000;18(3):353355.

16. Ballard SA, Gingell CJ, Tang K, Turner LA, Price ME, Naylor AM. Effects of sildenafil on the relaxation of human corpus cavernosum tissue in vitro and on the activities of cyclic nucleotide phosphodiesterase isoenzymes. J Urol. 1998; 159(6):2164-2171.

17. Lue TF. Erectile dysfunction. N Engl J Med. 2000;342(24): 1802-1813.

18. Mittleman MA, Maclure M, Glasser DB. Evaluation of acute risk for myocardial infarction in men treated with sildenafil citrate. Am J Cardiol. 2005;96(3):443-446. 
19. Ishikura F, Beppu S, Hamada T, Khandheria BK, Seward JB, Nehra A. Effects of sildenafil citrate (Viagra) combined with nitrate on the heart. Circulation 2000;102(20):25162521.

20. Herrmann HC, Chang G, Klugherz BD, Mahoney PD. Hemodynamic effects of sildenafil in men with severe coronary artery disease. N Engl J Med. 2000;342(22):16221626.

21. Cohen JS. Should patients be given an initial low test dose of sildenafil?. Drug Safety. 2000;23(1):1-9.

22. Arora RR, Timoney M, Melilli L. Acute myocardial infarction after the use of sildenafil (letter). N Engl J Med. 1999; 341(9):700.

23. Cheitlin MD, Hutter AM Jr, Brindis RG, Ganz P, Kaul S, Rusell RO, Zusman RM. Use of sildenafil (Viagra) in patients with cardiovascular disease (ACC/AHA Expert Consensus Document). Circulation 1999;99(1):168-177.

24. Halcox JPJ, Nour KRA, Zalos G, Mincemoyer R, Waclawiw MA, Rivera CE, et al. The effect of sildenafil on human vascular function, platelet activation, and myocardial ischemia. JACC 2002;40(7):1232-1240.
25. Salloum F, Yin C, Xi L, Kukreja RC. Sildenafil induces delayed preconditioning through inducible nitric oxide synthase-dependent pathway in mouse heart. Circ Res. 2003;92(6):595-597.

26. Shakir SAW, Wilton LV, Boshier A, Layton D, Heeley E. Cardiovascular events in users of sildenafil: results from first phase of prescription event monitoring in England. BMJ 2001;322(7287):651-652.

27. Wysowski DK, Farinas E, Swartz L. Comparison of reported and expected deaths in sildenafil (Viagra) users. Am J Cardiol. 2002;89(11):1331-1334.

28. Kekilli M, Beyazit Y, Purnak T, Dogan S, Atalar E. Acute myocardial infarction after sildenafil citrate ingestion. Ann Pharmacother. 2005;39(7-8):1362-1364.

Correspondencia autor: Dr. J.G. Velásquez López. Servicio de Urología. Hospital Pablo Tobón Uribe. Calle 78B, nº 69-240. 3466 Medellín - Colombia.

E-mail autor: juangvl@gmail.com Información artículo: Nota clínica (Trabajo aceptado abril 2006) 